\title{
TAGUNG
}

\section{Die Europäische Union als Akteur in der Arktis? Interessen und Regulierungsherausforderungen}

\author{
Arno Engel, Stefanie Mnich und Lisa Oberländer*
}

Die Zeiten, in denen die Arktis nur durch Errungenschaften naturwissenschaftlicher Forschung von sich reden machte, sind längst vorüber. Mehr denn je treffen dort verschiedene wirtschaftliche und politische Interessen aufeinander. Die Klimaerwärmung und das damit einhergehende Abschmelzen arktischen Eises haben nicht nur Auswirkungen für die Völker und Volkswirtschaften des Hohen Nordens, sondern sind auch für andere Staaten von großer Bedeutung. Im Hinblick auf arktische Rohstoffe und neue Handelsrouten ist bei einigen Wirtschaftsakteuren eine regelrechte Goldgräberstimmung festzustellen, die einen der Gründe dafür darstellt, dass auch die Europäische Union eine aktivere Rolle in der Region anstrebt. Ziel der dritten Geopolitics in the High North (GEONOR) Konferenz war es, Rollenzuschreibungen und effektiv wahrgenommene Funktionen der Europäischen Union zu untersuchen. Darüber hinaus befasste sich die Konferenz mit der Frage, inwiefern und wann eine Verwirklichung des ökonomischen Potenzials der Arktis tatsächlich möglich erscheint.

Als bedeutender Markt für arktisches Öl und Gas sowie Fisch aus der Region ist die Europäische Union ein erheblicher Wirtschaftsfaktor im Hohen Norden. Als Handelsmacht verspricht sie sich langfristig kürzere und sicherere Seerouten durch den zunehmend frei tauenden Arktischen Ozean. Auch für die europäische Industrie, die über bedeutende Expertise in den für die widrigen Bedingungen notwendigen Technologien verfügt, könnte eine

\section{,The EU as an Arctic Actor? Interests and Governance Challenges"“}

3rd Annual Geopolitics in the High North International Conference

Organisiert von der Stiftung Wissenschaft und Politik

Berlin, 22.-24. Mai 2012

Begrüßung und Dinner Speech

Sven Erik SVEDMAN, Norwegischer Botschafter, Berlin

\section{Begrüßung und Einführung}

Dr. Barbara LIPPERT, Forschungsdirektorin, Stiftung Wissenschaft und Politik, Berlin

Prof. Dr. Rolf TAMNES, Direktor, Norwegian Institute for Defence Studies, Oslo

Die Europäische Union als Akteur in der Arktis? Interessen und Governance-Herausforderungen

Vorsitz

Dr. Barbara LIPPERT, Forschungsdirektorin, Stiftung Wissenschaft und Politik, Berlin

Dr. Andreas MAURER, Senior Fellow, Stiftung Wissenschaft und Politik, Brüssel

Kurzstatements

Claus GRUBE, Staatssekretär, Königlich Dänisches Außenministerium, Kopenhagen

Karsten KLEPSVIK, Sonderberater für polare Angelegenheiten, Norwegisches Außenministerium, Oslo

MinDirig Stephan AUER, Beauftragter für Globalisierung, Energie- und Klimapolitik, Auswärtiges Amt, Berlin

* Arno Engel, Master-Student in International Public Management, Sciences Po, Paris.

Stefanie Mnich, Master-Studentin der Skandinavistik/Nordeuropastudien, Humboldt Universität zu Berlin.

Lisa Oberländer, Master-Studentin der Volkswirtschaftslehre, Ruprecht-Karls-Universität, Heidelberg. 
erhöhte wirtschaftliche Aktivität im Hohen Norden zu neuen Aufträgen führen. Auf der anderen Seite haben die hohen Treibhausgasemissionen der Europäischen Union einen starken Anteil am für die Arktis folgenreichen Klimawandel, für den sie sich auch in der Verantwortung sieht.

\section{Eine Rolle für die Europäische Union im Hohen Norden: zwischen ökonomischen Interessen und Regulierungsverantwortung}

Die unterschiedlichen Interessen der verschiedenen Akteure in der Arktis und daraus resultierende politische Herausforderungen waren Gegenstand der ersten Sitzung. Seit einiger Zeit sei man sich in der Wissenschaft weitgehend darüber einig, dass sich die klimatischen Bedingungen im Hohen Norden verändern. Die Auswirkungen dieses Temperaturanstiegs seien vielfältig und verursachten unter anderem das Abschmelzen der nördlichen Polarkappe. Auch wenn die daraus resultierenden Folgen für Mensch und Umwelt heute noch nicht exakt zu bestimmen seien, herrsche Einigkeit darüber, dass die Auswirkungen auch über die Region hinaus, wenn nicht sogar global, spürbar sein werden.

Diese fortschreitende Eisschmelze sei nicht nur wissenschaftlich bedeutsam, sondern eröffne zudem neue wirtschaftliche Möglichkeiten, da sich 10 Prozent der weltweit unentdeckten Ölvorkommen und 30 Prozent der weltweit unentdeckten Gasvorkommen in der Arktis befänden. Allerdings lagerten 90 bis 95 Prozent aller arktischen Rohstoffvorkommen in den Ausschließlichen Wirtschaftszonen der arktischen Staaten, weshalb ihr Abbau größtenteils nationalen Gesetzgebungen unterliege. Auch könne sich die Schifffahrt in der Arktis zu einem rentablen Wirtschaftszweig entwickeln, da die künftig wahrscheinlich befahrbaren Passagen wichtige Handelsrouten signifikant verkürzten, im Gegensatz zum Horn von Afrika, dem Golf von Aden, und den Straßen von Malakka und Singapur sicherer seien und sich zusätzlich Chancen für den Tourismus böten.
Bernhard FRIESS, Europäische Kommission, Direktor, Direktorat für den Atlantik, Regionen in äußerster Randlage und die Arktis, Generaldirektion Maritime Angelegenheiten und Fischerei, Brüssel Dr. habil. Volker RACHOLD, Executive Secretary, International Arctic Science Committee (IASC) und Alfred Wegener Institute für Polar- und Meeresforschung, Potsdam

Governance in der Arktis und die Rolle der Europäischen Union und ihrer Mitgliedstaaten Vorsitz

Steffen WEBER, Generalsekretär, EU Arctic Forum, Brüssel

\section{Kurzstatements}

Dr. Michael BRAVO, Senior Lecturer, Department of History and Philosophy of Science, University of Cambridge

Dr. Andreas MAURER, Stiftung Wissenschaft und Politik, Brüssel

Stewart ARNOLD, Policy and Communications Adviser, Europäisches Parlament, Brüssel

Kommentar

Prof. Dr. Oran R. YOUNG, Bren School of Environmental Science and Management, University of California, Santa Barbara

Natürliche Ressourcen in der Arktis: Interessen der Europäischen Union und Regulierungsherausforderungen

Vorsitz

Dr. Kristine OFFERDAL, Programme Manager, Geopolitics in the High North, Norwegian Institute for Defence Studies (IFS), Oslo

Kurzstatements

Dr. Christian REICHERT, Leiter der Unterabteilung Marine Rohstofferkundung, Bundesanstalt für Geowissenschaften und Rohstoffe, Hannover Jonas GRÄTZ, Forscher, Center for Security Studies, Eidgenössische Technische Hochschule Zürich

Robert BLAUUW, Leiter, Global Arctic Theme, Royal Dutch Shell, Den Haag

Andreas ØSTHAGEN, The Arctic Institute - Center for Circumpolar Security Studies, Washington D.C. / North Norway European Office, Brüssel Dr. Stefan MAIR, Mitglied des Vorstands, Bundesverband der Deutschen Industrie e.V., Berlin

Kommentar

Dr. Andreas MAURER, Stiftung Wissenschaft und Politik, Brüssel 
Die wirtschaftliche Erschließung des Hohen Nordens gehe jedoch mit großen Risiken für die Umwelt einher. Gefahren wie ein verstärkter Kohlendioxidausstoß oder Ölkatastrophen müsse begegnet werden. Da jede Veränderung des arktischen Ökosystems globale Auswirkungen haben werde, seien Kooperationen auf internationaler Ebene notwendig. Zurzeit reichten die Kapazitäten jedoch nicht, um diesen Risiken effektiv begegnen zu können, weshalb alle arktischen Staaten zu einer aktiveren Mitarbeit beim Umweltschutz angehalten seien.

Der Arktische Rat nehme hierbei als hochrangiges zwischenstaatliches Forum für die Kooperation, Koordination und Interaktion der arktischen Staaten eine Schlüsselrolle ein und habe sich der Förderung nachhaltiger Entwicklung sowie dem Umweltschutz verschrieben. Die Europäische Union sei nur durch einzelne Mitgliedstaaten im Arktischen Rat vertreten, deren Mitglieder unterschiedliche Positionen zu dem von der Europäischen Union geäußerten Wunsch nach einem ständigen Beobachterstatus einnähmen. Als Hauptgrund für diesen Antrag führe die Europäische Union ihre Rolle als Schadstoffemittent an, da ihre 500 Millionen Bürger für 40 Prozent aller Emissionen in der Arktis verantwortlich seien. Die Europäische Union fungiere darüber hinaus als ein wichtiger Akteur in der arktischen Fischerei und engagiere sich stark im Bereich der Forschung. So investierte sie 200 Millionen Euro im Rahmen ihres siebten Rahmenforschungsprogramms für arktische Forschungsprojekte und unterstütze zudem soziale und wirtschaftliche Projekte für die Menschen in der Arktis über ihre regionalen Förder- und Kooperationsprogramme. Sie sehe sich in einer den Arktischen Rat unterstützenden und mit ihm kooperierenden Rolle und sei auf dem Weg, zu einem großen, wenn nicht sogar dem größten Förderer der arktischen Forschung zu werden.

Bei diesem Annäherungsprozess sehe sich Dänemark in einer vermittelnden Rolle, da es sowohl ein Mitgliedstaat der Europäischen
Umweltschutz: Interessen der Europäischen Union und Governance-Herausforderungen Vorsitz

Antje NEUMANN, Fellow, Forschungsgruppe EU-Integration, Stiftung Wissenschaft und Politik, Berlin

Kurzstatements

Prof. Dr. Timo KOIVUROVA, Leiter des Northern Institute for Environmental and Minority Law, Arctic Centre, Universität Lapland, Rovaniemi Prof. Dr. Olav SCHRAM STOKKE, Research Professor, Fridtjof Nansen Institute, Oslo

Dr. Bettina RUDLOFF, Senior Fellow, Forschungsgruppe EU-Außenbeziehungen, Stiftung Wissenschaft und Politik, Berlin

Kommentar

Dr. Rasmus Ole RASMUSSEN, Senior Research Fellow, Nordregio, Brüssel

Schifffahrt und Transport: Interessen der

Europäischen Union und Governance-

Herausforderungen

Vorsitz

Dr. Bettina RUDLOFF, Stiftung Wissenschaft und Politik, Berlin

Kurzstatements

Prof. Dr. Erik MOLENAAR, Netherlands Institute for the Law of the Sea (NILOS), Utrecht

Josep CASANOVAS, Transport Policy Unit, Generaldirektion Mobilität und Verkehr, Europäische Kommission, Brüssel

Burghard ZIMMERMANN, Leiter Konstruktion, Nordic Yards, Wismar

Kommentar

Andreas RASPOTNIK, Analyst, The Arctic Institute - Center for Circumpolar Security Studies, Brüssel

Die sicherheitspolitischen Entwicklungen in der Arktis: Spielraum für Kooperationen?

Vorsitz

Oberst (GS) Rainer MEYER ZUM FELDE, Vizepräsident, Bundesakademie für Sicherheitspolitik (BAKS), Berlin

Kurzstatements

Prof. Dr. Clive ARCHER, Manchester Metropolitan University, Manchester

Prof. Dr. Carlo MASALA, Fakultät für Staats- und Sozialwissenschaften, Universität der Bundeswehr München 
Union als auch ein Mitglied des Arktischen Rates sei, und betone die Notwendigkeit, den Umweltschutz bei der wirtschaftlichen Erschließung der Region zu berücksichtigen. Deutschland unterstütze das Streben der Europäischen Union nach einem Sitz als ständiger Beobachter im Arktischen Rat und unterstreiche dessen Schlüsselrolle für arktische Angelegenheiten. Diesem böte es daher seine volle Unterstützung für alle anstehenden Herausforderungen an und verweise dabei vor allem auf seine technische Expertise beim Bau von Eisbrechern und von an die rauen arktischen Bedingungen angepassten Schiffen.

\section{Nationale Interessen und die Zukunft der Arktispolitik der Europäischen Union}

Das zweite Panel thematisierte die gegenwärtige und zukünftige Rolle der Europäischen Union und ihrer Mitgliedstaaten bei der Bewältigung von Regulierungsproblemen in der Arktis. Unterstrichen wurde dabei insbesondere der Einfluss, den verschiedene Diskurse (Narrative) über arktische Governance-Probleme auf die politischen Rahmenbedingungen haben. Dabei wurden folgende Hauptdiskurse identifiziert: Ein geopolitischer Diskurs, der sicherheitspolitische Aspekte betone, ein geoökonomischer Diskurs mit Fokus auf Ressourcen und Transport sowie ein geoökologischer Diskurs, der besonders die Rolle des Klimawandels berücksichtige. Die Handlungen und Entscheidungen politischer Akteure und Staaten und somit die zukünftige Entwicklung im Hohen Norden werde von eben solchen verschiedenen Denkmustern maßgeblich beeinflusst. Zurzeit werde die geoökonomische Perspektive aufgrund der intensiven Diskussion über das Potenzial neu entstehender Handelsrouten und die Förderung von insbesondere fossilen Rohstoffen als dominant angesehen. Sollte jedoch der geopolitische Diskurs an Bedeutung gewinnen, würde auch die Gefahr von Konflikten steigen.

Doch auch der gegenwärtig dominierende Fokus auf Ressourcen berge bereits Risiken, da andere wichtige Aspekte unzureichend beach-
Dipl.-Pol. Stefan STEINICKE, Forschungsassistent, Geopolitics in the High North, Stiftung Wissenschaft und Politik, Berlin

Kommentar

Prof. Dr. Helga HAFTENDORN, Freie Universität Berlin

\section{Abschlusspanel}

Vorsitz

Dr. Andreas MAURER, Stiftung Wissenschaft und Politik, Brüssel

Kurzstatements

Steffen WEBER, Generalsekretär, EU Arctic Forum, Brüssel

Antje NEUMANN, Stiftung Wissenschaft und Politik, Berlin

Dr. Bettina RUDLOFF, Stiftung Wissenschaft und Politik, Berlin

Dr. Kristine OFFERDAL, Norwegian Institute for Defence Studies, Oslo

tet würden. So gelte es beispielsweise, das jeweilige Autonomieverständnis der einzelnen Völker und Akteure im arktischen Raum zu verstehen um eine bessere Kooperation zu ermöglichen. Dies sei auch für das Bestreben der Europäischen Union, den Beobachterstatus im Arktischen Rat zuerkannt zu bekommen, relevant. Überdies sei es wichtig, die gesamte Bandbreite der derzeitigen Entwicklungen in der Region zu verstehen. Neue Kapitalzuflüsse seien beispielsweise ungleich verteilt und führten zu einem sozioökonomischen Gefälle. Die geografische Lage von und der Zugang zu Ressourcen verändere bestehende Machtgefüge und die Entwicklung der Schifffahrt beeinflusse die Infrastrukturplanung sowie die ökonomische Lebensgrundlage der lokalen Bevölkerung.

Diese verschiedenen Aspekte seien auch für die Arktispolitik der Mitgliedstaaten und der Europäischen Union selbst entscheidend. Mit der Norderweiterung habe die Europäische Union bereits in den 1990er Jahren die Grundlage für die Politik der nördlichen Dimension gelegt. Heute verfolge sie vielfältige Ziele und unterstütze unter anderem die Entwicklung 
multilateraler Regulierungsstrukturen für die Arktis. In ihren Beziehungen zu Russland stehe die Europäische Union der Herausforderung gegenüber, sowohl den eigenen Werten als auch der Interdependenz im Bezug auf natürliche Ressourcen Rechnung tragen zu müssen. Auf der anderen Seite müsse die Union ihr Verhältnis zu den USA justieren, die in ihrer Arktispolitik den sicherheitspolitischen Aspekten größere Bedeutung beimessen.

Eine große Herausforderung für die Rolle der Europäischen Union bestehe in ihrem wenig geschlossenen Auftritt in arktischen Angelegenheiten. Als Vertreter Grönlands und der Färøer-Inseln sei Dänemarks Position nicht immer im Einklang mit derjenigen der Europäischen Union und behindere die Entwicklung eines konsistenten Ansatzes. Von allen arktischen Staaten würde Finnland am pointiertesten eine starke Rolle der Europäischen Union befürworten und versuche, europäische Politiken vor Ort zu implementieren. Auch ein EU-Beitritt Islands könnte zu einer stärkeren Position der Union führen. Deutschland und Schweden bemühten sich überdies um eine Vermittlerrolle zwischen den Arktisstaaten und der Union.

Aus dem Fehlen einer konsistenten Arktispolitik erwachse jedoch nicht die Notwendigkeit einer europäischen Arktisstrategie. Ein solches Dokument würde vielmehr Erwartungen wecken, die aufgrund des geltenden Kompetenzgefüges innerhalb der Europäischen Union nicht erfüllt werden könnten. Daher solle sich die Europäische Union vor allem um eine größere Kohärenz ihrer verschiedenen Politiken bemühen und auf (bestehende) internationale Abkommen wie etwa im Rahmen der Internationalen Seeschifffahrtsorganisation oder der Welthandelsorganisation zurückgreifen und zu deren Weiterentwicklung beitragen.

\section{Die Arktis als Rohstofflieferant: Interessen der Europäischen Union und politische Herausforderungen}

Das dritte Panel diskutierte die Interessen der Europäischen Union an natürlichen Ressour- cen in der Arktis und die damit verbundenen politischen Herausforderungen. Zunächst wurde betont, dass die Arktis sowohl im Bereich der Energieressourcen und Metalle als auch bei lebenden, biologischen Ressourcen über großen Reichtum verfüge. Schwankende Rohstoffpreise und der voraussichtlich bessere $\mathrm{Zu}$ gang seien die Hauptgründe für das zunehmende Interesse europäischer Akteure an arktischen Ressourcen. Die meisten Rohstoffe selbst lägen jedoch innerhalb der ausschließlichen Wirtschaftszonen der Anrainerstaaten, auch wenn sich möglicherweise aus wissenschaftlicher Perspektive Argumente für eine Ausdehnung des Bereichs unter internationaler Kontrolle finden ließen.

Allerdings würden die Herausforderungen für die Ressourcenförderung in der Arktis voraussichtlich erst in 20 bis 30 Jahren überwunden sein, sodass sie noch keine sichere Investition darstelle. Zudem sei die Entwicklung der arktischen Rohstoffförderung von Logistik und Transportmöglichkeiten abhängig. Aus regulatorischer Sicht bestünden jedoch seit der Respektierung des Seerechtsübereinkommens der Vereinten Nationen (SRÜ) durch die USA keine größeren Unstimmigkeiten zwischen den fünf arktischen Staaten mehr. Der Europäischen Union bliebe in der Arktis politisch nur die Rolle eines Wegbereiters; im Gegensatz zu ihren Mitgliedstaaten und dem privaten Sektor könne sie nicht direkt eingreifen.

Als weltweit größter Importeur von Energie sollte für die Europäische Union die Diversifizierung ihrer Bezugsquellen ein Hauptziel der Energiepolitik darstellen. Eine enge Partnerschaft mit Norwegen, dem zweitgrößten Gasexporteur der Welt, sei dabei von strategischer Bedeutung, zumal die norwegische Wirtschaft nicht nur in der Technologie für die Offshore-Förderung von Öl und Gas Maßstäbe setze, sondern auch die hohen Umweltstandards erfüllen könne. Die Europäische Union solle daher die norwegische Verwaltung Spitzbergens stärken, denn zurzeit sei unklar, ob die Inselgruppe den Status einer Wirtschaftszone besitze und ein Rohstoffabbau in Zukunft mög- 
lich sei. Für einen größeren EU-Einfluss sollten die Mitgliedstaaten einen gemeinsamen Standpunkt bei Streitigkeiten um Energieressourcen und Gebiete in der Arktis formulieren. Strittig blieb jedoch die Frage, ob die Europäische Union angesichts des demografischen Wandels sowie ihrer internen Energiestrategien, die vor allem auf die Förderung der erneuerbaren Energien abzielen, auch in Zukunft einen wichtigen Energiemarkt für arktische Exportnationen darstelle.

Während Kanada seine Gasressourcen aufgrund unzureichender Infrastruktur bis jetzt nicht gefördert habe, fungiere Russland als größter Gasexporteur der Welt und „Hauptversorger“ der Europäischen Union. Russland verfüge über eine der größten Gasressourcen der Erde und werde auch zukünftig der dominante arktische Akteur im Energiesektor sein. Ausländische Investoren könnten in der russischen Arktis allerdings nur einmalige Abmachungen treffen. Auf diese Weise stelle Russland technologische Fähigkeiten und Risikokapital sicher und unterstütze damit heimische Firmen, während ausländische Firmen ein hohes Risiko trügen. Nicht nur die Einführung von Regeln für höhere Umweltstandards sei in Russland problematisch, auch würden bereits eingeführte Regeln ignoriert, wenn sie dem „nationalen Interesse“ entgegenstünden.

Aus Sicht der Industrie sei eine verantwortungsbewusste Entwicklung der arktischen Region möglich, mit deren Rohstoffen 15 Prozent des globalen Energiebedarfs gedeckt werden könnten. Lokale Kommunen erhielten auf diese Weise Entwicklungsmöglichkeiten. Die Entscheidung einiger Arktisanrainer, die Offshore-Förderung von Ö1 und Gas zu genehmigen, werde begrüßt, dabei müsse jedoch auf Nachhaltigkeit Wert gelegt werden und die sozioökonomische Struktur und Kultur der indigenen Bevölkerung respektiert werden.

Im Interesse der deutschen Industrie liege vor allem eine zuverlässige, nachhaltige und bezahlbare Energieversorgung. Im Zuge von Ressourcen-Allianzen wolle die deutsche In- dustrie den langfristigen Bedarf decken, dabei werde das Potenzial des Hohen Nordens aber bisher weitestgehend ignoriert.

\section{Umweltschutz in der Arktis: Welche Rolle für die Europäische Union?}

Der Umweltschutz in der Arktis stand im Zentrum des vierten Panels, das insbesondere der Frage nachging, wie die Europäische Union sich stärker für den Schutz der Umwelt im Hohen Norden einsetzen könnte und welche Vorteile sich hieraus für sie ergäben.

Die Notwendigkeit einer verstärkten Präsenz der Europäischen Union wurde zunächst jedoch infrage gestellt, da nach Auffassung einiger Wissenschaftler die von einzelnen arktischen Staaten sowie die im Arktischen Rat entwickelten Ansätze zum Schutz des arktischen Biosystems ausreichten.

Allerdings werde die Arktis auch durch Einflüsse geschädigt, deren Ursprünge außerhalb der Region lägen. Als Beispiele könnten der Klimawandel sowie die Verschmutzung der Arktis mit langlebigen organischen Schadstoffen und Schwermetallen angeführt werden. Regionale Lösungsansätze seien angesichts dieser globalen Einflüsse als unzureichend anzusehen; arktische Probleme bedürften daher auch „nicht-arktischer“ Lösungen. Somit sei ein stärkeres Engagement der Europäischen Union durchaus zu rechtfertigen, zumal dies auch andere Akteure dazu veranlassen könnte, sich ebenfalls stärker für die Umweltbelange in der Arktis einzusetzen. Zwar sei die Gesetzgebung der Europäischen Union in weiten Teilen der Arktis nicht anwendbar. Bei einer konsequenten Anwendung ihrer strengen Regelungen zum Schutz internationaler Gewässer könne sie jedoch einen wichtigen Beitrag bei der Strafverfolgung innerhalb der europäischen Staatengemeinschaft leisten.

Die unsystematischen Ansätze der Europäischen Union zum Schutz des arktischen Lebensraums hätten ihre Ursachen in der komplexen Kompetenz- und Organisationsstruktur 
sowie in unterschiedlichen, teils erheblich divergierenden Interessen einzelstaatlicher Akteure innerhalb der Europäischen Union. Positiv zu bewerten sei jedoch, dass die Europäische Union bei den zurückliegenden Quecksilberverhandlungen im Rahmen des Umweltprogramms der Vereinten Nationen geschlossen und intensiv für eine verbindliche Übereinkunft geworben habe. Auch im Kampf gegen die globale Erwärmung nehme die Europäische Union durch ihre Selbstverpflichtung zur Reduzierung der Rußemissionen eine Vermittlerrolle bei den internationalen Verhandlungen ein. Auf der Suche nach einem identitätsstiftenden Platz in der internationalen Staatengemeinschaft strebe die Europäische Union eine globale Führungsrolle beim Schutz der Umwelt an. Gestalte sie ihren Politikansatz zukünftig systematischer und politikbereichsübergreifend kohärenter, könne sie dank der Kombination aus Ehrgeiz und regionalem Gewicht tatsächlich eine progressive Rolle beim Schutz der arktischen Umwelt spielen. Ein Sitz als ständige Beobachterin des Arktischen Rates wäre diesem Ziel sicherlich förderlich.

Abgesehen von diesen Bemühungen auf globaler Ebene, böten sich für die Europäische Union auch Möglichkeiten zur Einflussnahme auf regionaler Ebene. Dies gelte besonders für die Fischerei, da der europäische Binnenmarkt für die arktische Fischereiindustrie den wichtigsten Absatzmarkt darstelle und sie somit über große Marktmacht verfüge. Der Fischbestand in der Arktis sei schwer zu erforschen und werde zudem durch den Klimawandel in unvorhersehbarer Weise beeinflusst. Daraus sowie aus einer stärkeren Bewirtschaftung des arktischen Fischbestandes könnten unvorhersehbare Konsequenzen für das gesamte Ökosystem resultieren. Die Fischereiindustrie ist in regionalen Fischereiorganisationen organisiert, die sich um die Fischbestände bestimmter
Gebiete oder auch bestimmter Spezies kümmern. Es existierten jedoch einige geografische Lücken in diesem Managementsystem, welche für eine nachhaltige Bewirtschaftung der arktischen Fischgründe unbedingt zu schließen seien. Als ein möglicher Lösungsansatz sei hierbei eine geografische Ausweitung bestehender regionaler Fischereiorganisationen, möglichst unter Berücksichtigung der ökosystemischen Struktur (Ökosystemansatz), denkbar. Eine stärkere Bekämpfung der illegalen, unregulierten und ungemeldeten Fischerei (IUU-Fischerei), die Abkehr von der Praxis, unerwünschte Beifänge zurück ins Meer zu werfen sowie eine generelle Reduzierung der Fangquoten wurden als weitere Vorschläge genannt, wie die Europäische Union nachhaltige Fischerei in der Arktis fördern könne. Schließlich wurde auch auf die lokale Ebene beim Schutz der arktischen Umwelt verwiesen, wobei insbesondere die Interessen der einheimischen Bevölkerung berücksichtigt werden sollten.

\section{Arktische Schifffahrt - Eine Chance für die Europäische Union?}

Im fünften Panel wurden rechtliche, politische und wirtschaftliche Aspekte der Schifffahrt in arktischen Gewässern diskutiert sowie die Rolle der Europäischen Union in diesem Sektor. Trotz insgesamt positiver Zukunftsaussichten für die Schifffahrt in der Region stellten die Podiumssprecher fest, dass in nahezu allen Bereichen noch viel Klärungsbedarf bestehe und Entwicklung nötig sei, bevor das ökonomische Potenzial verwirklicht werden könne. Als zentrale Dokumente für die europäische Arktispolitik wurden in diesem Zusammenhang die Mitteilung der Kommission ${ }^{1}$ und die Resolution des Europäischen Parlaments ${ }^{2}$ aus dem Jahre 2008 sowie die kurze Zeit nach der Kon-

1 Kommission der Europäischen Gemeinschaften: Mitteilung der Kommission an das Europäische Parlament und den Rat. Die Europäische Union und die Arktis, KOM (2008) 763.

2 Europäisches Parlament: Politisches Handeln im arktischen Raum in einer Welt der Globalisierung. Entschließung des Europäischen Parlaments vom 9. Oktober 2008 zu der Politik für den arktischen Raum, in: Amtsblatt der EU, Nr. C 9 E vom 15. Januar 2010, S. 41-43. 
ferenz veröffentlichte neue Mitteilung der Kommission von 2012 genannt. $^{3}$

Als Nicht-Anrainer des Arktischen Ozeans besäße die Europäische Union keine besonderen rechtlichen Mittel, um ihre Interessen geltend zu machen. Jedoch sei es Mitgliedstaaten möglich, über ihre Häfen, Ausbildungs- und Sicherheitsstandards für Seeleute und die unter ihrer Fahne segelnden Schiffe bestimmte Regelungen durchzusetzen. Außerdem könnten Staaten wie zum Beispiel Finnland ihre umfassende Erfahrung in arktischer Schifffahrt einbringen. Auf außenpolitischer Ebene bestünden in den Beziehungen zu Russland und Kanada unterschiedliche Rechtsauffassungen bezüglich des Status der an den jeweiligen Küsten verlaufenden Seerouten (die Nordostund die Nordwestpassage). Während die Anrainerstaaten diese als Teil ihrer ausschließlichen Wirtschaftszone unilateral regelten, forderten Handelsmächte in Europa und die USA implizit oder explizit diese Routen als Transitdurchfahrt gemäß dem SRÜ anzusehen und Schiffen somit eine freie und unbehelligte Durchfahrt zu garantieren. Mit dem Verweis auf die Einigung im Territorialstreit zwischen Norwegen und Russland wurde jedoch die Erwartung geäußert, dass auch in Zukunft Grund für Optimismus bezüglich der Lösung von Konflikten bestünde.

In ihren Bemühungen um multilaterale Regelungen für die Arktis unterstütze die Europäische Union zum Beispiel das SRÜ als rechtlichen Rahmen und trage als Koordinator zur Arbeit innerhalb der Internationalen Seeschifffahrtsorganisation und zur Entwicklung eines Polarkodexes für die Schifffahrt bei. Überdies sei die Europäische Agentur für die Sicherheit des Seeverkehrs (EMSA) in der Region tätig, die auch von über einer Milliarde Euro an Struktur- und Regionalfonds profitiere. Des Weiteren fördere die Union arktische Forschungsprojekte und setze sich für eine nach- haltige Ressourcennutzung ein. Als bedeutender Akteur im Seehandel besitze die Union ein großes Interesse an einer Ausweitung der arktischen Schifffahrt, insbesondere, da einige der zukünftig frei werdenden Routen bis zu 40 Prozent kürzer sein könnten als die derzeit befahrenen Strecken. Somit könnten Zeitersparnisse realisiert sowie Kosten und Emissionen gesenkt werden.

Die derzeitige und zukünftige Entwicklung der arktischen Schifffahrt werde einerseits angetrieben von den (vermuteten) natürlichen Ressourcen in der Region, steigendem Interesse an Kreuzfahrten, der Hoffnung auf kürzere Routen, aber auch von dem technischen Fortschritt, der die Kosten für die benötigte Ausrüstung senken könnte. Allerdings sehe sich die arktische Schifffahrt nach wie vor großen Herausforderungen gegenüber. So fänden zwar zur Versorgung der Inuitbevölkerung, zum Transport von Rohstoffen aus der Region, für den Tourismus und zu wissenschaftlichen Zwecken regelmäßig Fahrten in der Region statt; Transitfahrten hätten jedoch bisher keine ökonomische Bedeutung. Aufgrund der großen Anforderungen an Material und Personal, die durch das Eis, die lange Dunkelheit und die Entlegenheit der Routen erwachsen, könne die Schifffahrt nur in Nischenmärkten, wie etwa dem Transport besonderer Metalle aus der Region, rentabel sein. Auch wenn die Industrie ein breites Angebot an arktistauglichen Schiffen herstellen könne, bleibe die Rentabilität eine große Herausforderung für die zukünftige Entwicklung. Daher hoffe die Industrie, dass sich ein Szenario geprägt von hoher Nachfrage nach Handel und Ressourcen, einer zuverlässigen Regulierung und einem nachhaltigen Umgang mit der Umwelt einstelle.

\section{Sicherheitspolitik in der Arktis: Konflikt- potenziale und Kooperationsmöglichkeiten}

In der sechsten Sitzung diskutierten die Teilnehmer über die sicherheitspolitischen Ent-

3 Europäische Kommission/Hohe Vertreterin der Europäischen Union für Außen- und Sicherheitspolitik: Gemeinsame Mitteilung an das Europäische Parlament und den Rat. Entwicklung einer Politik der Europäischen Union für die Arktis: Fortschritte seit 2008 und nächste Schritte, JOIN (2012) 19. 
wicklungen in der Arktis. Zu den immer noch präsenten harten Sicherheitsbelangen zählten das Hissen der russischen Flagge auf dem Meeresboden des Nordpols 2007 sowie die militärische Präsenz Russlands in der Region. Offene bilaterale Streitigkeiten bestünden zwischen den USA und Kanada, Dänemark und Kanada sowie den USA und Russland. Auch die Nichtratifizierung des SRÜ durch die USA sowie der Zugang zu neu entstehenden Seerouten bergen Konfliktpotenzial. Zudem sei die Rolle der NATO in der Arktis umstritten. Neue Ressourcen- und Transportfragen beträfen sowohl harte als auch weiche Sicherheitsaspekte. Zu Letzteren zählten auch der große Ölbesitz eines einzigen Staates und die neuen Möglichkeiten für den Tourismus. Gefahren entstünden durch Terrorismus, Verschmutzung, Erderwärmung und Unfälle. Die Konsequenzen träfen die indigene Bevölkerung, die ihren Lebensstil anpassen müsse, aber auch europäische maritime Staaten wie Großbritannien, Dänemark und die Niederlande.

Die Hauptakteure in der Arktis seien die im Arktischen Rat kooperierenden Staaten, die fünf Anrainerstaaten (A-5) sowie die Europäische Union, China und Japan. Die wichtigsten nichtstaatlichen Akteure seien die indigene Bevölkerung, Nichtregierungsorganisationen sowie Firmen, die in der Arktis wirtschaftlich aktiv seien. Die aufgezeigten Konfliktlinien, sowohl im Bereich der harten als auch der weichen Sicherheitspolitik, zeugten von der Notwendigkeit intensiver Kooperation. Als Instrumente der Zusammenarbeit stünden hierfür das SRÜ, die Internationale Seeschifffahrtsorganisation, die Euroarktische Barentsregion, der Euroarktische Barentsrat, die Europäische Union mit der Nördlichen Dimension und Arktis-Politik, die NATO, der Arktische Rat, der Nordische Rat und der Nordische Ministerrat zur Verfügung. Diese Vielzahl internationaler Foren unterstreiche den Stellenwert multilateraler Institutionen für den politischen Dialog über die Arktis, im Vergleich mit dem Instrument der bilateralen Kooperationen, wie sie zum Beispiel zwischen Norwegen und Russland sowie Norwegen und Kanada existieren.
Im Hinblick auf künftige Governance-Entwicklungen in der Arktis wurden zwei Szenarien diskutiert: Im ersten seien Ressourcen durch den einfacheren Zugang für verschiedene Akteure Anlass für einen nationalen Wettkampf, wodurch die Militarisierung der Arktis zunähme. Im zweiten Szenario befänden sich Ressourcen maßgeblich unter nationaler Kontrolle und die verschiedenen Akteure arbeiteten im Rahmen von Institution kooperativ zusammen, auch im Hinblick auf die Umwelt und wirtschaftliche Entwicklung.

Daran anknüpfend wurde mit Blick auf zukünftige Handlungsmöglichkeiten der Akteure die These formuliert, dass die Europäische Union keine Rolle in traditionellen Sicherheitsthemen in der Arktis einnehmen könne. Ihr böten sich jedoch zwei Möglichkeiten, um im Bereich der weichen Sicherheit zur Zusammenarbeit beizutragen: Zum einen sei hier das neue Satellitenprogramm Galileo zu nennen, welches auch unter sehr extremen Bedingungen einen Ortungsdienst anbieten könne, und zum anderen wurde auf die europäische „Global Monitoring for Environment and Security" Initiative verwiesen, die auch Daten zum Klimawandel generiere.

Bei einer genauen Betrachtung der Positionierung einzelner, nichtarktischer Mitgliedstaaten hinsichtlich arktischer Sicherheitsbelange ergebe sich jedoch ein differenzierteres Bild. Deutschland, Frankreich und Großbritannien hätten die strategische Bedeutung der Arktis im Hinblick auf wirtschaftliche und ökologische Sicherheit erkannt. Besonders Großbritannien bemühe sich um eine aktivere Rolle und unterstütze zusätzlich zu seinem Engagement im Northern Future Forum und seinen intensivierten bilateralen Beziehungen zu Norwegen und Kanada auch die Bildung einer nordischen Gruppe innerhalb der NATO. Innerhalb der Europäischen Union erachte mit Ausnahme von Finnland keiner der anderen Mitgliedstaaten die Gemeinsame Sicherheits- und Verteidigungspolitik als einen geeigneten Rahmen für Diskussionen über arktische Sicherheitsbelange. Die Möglichkeit, die Solidaritätsklausel des Vertrags von Lissabon im Fal- 
le einer Ölpest anzuwenden, werde nur von Frankreich und Großbritannien angesprochen. Im deutschen Diskurs existiere dagegen keine militärische Dimension der Arktis; eine solche mitzudenken sei für eine aktivere Vermittlerrolle Deutschlands jedoch förderlich.

Schließlich sei im Bezug auf die NATO als internationaler Akteur in der Region zu erwähnen, dass diese sicherlich keine militärischen Mittel in der Arktis einsetzen werde und zudem das russische Misstrauen gegenüber ihrer Präsenz im Hohen Norden ihren Handlungsradius begrenze. Möglicherweise könnte die NATO jedoch bei der Lösung weicher Sicherheitsprobleme in der Arktis durch technische Unterstützung oder der Einrichtung einer Küstenwache behilflich sein.

Eine erfolgreiche Arktispolitik der Europäischen Union erfordert Kohärenz zwischen wirtschaftichen und umweltpolitischen Zielen

Die Europäische Union hat als regionale Wirtschaftsmacht und großer Treibhausgasemittent ökonomischen sowie auch ökologischen Einfluss auf den Hohen Norden. Aus der sich stetig verbessernden Zugänglichkeit der Arktis erwachsen ökonomische Potenziale vor allem in den Bereichen Rohstoffförderung und Handelsrouten, allerdings birgt eine wirtschaftli- che Erschließung auch Konfliktpotenziale im Hinblick auf Klimapolitik und Umweltschutz. Das Interesse der Europäischen Union an der Region manifestiert sich am deutlichsten in ihrer starken Förderung arktischer Forschungsprojekte. Dieses Engagement könnte in der $\mathrm{Zu}$ kunft als ein wichtiger Baustein auf dem Weg zu einer allgemeinen Anerkennung ihrer Rolle als legitimierter politischer Akteur in der Arktis fungieren; der angestrebte Erhalt eines Sitzes als ständige Beobachterin im Arktischen Rat stellt hierbei ein wichtiges Etappenziel dar. Ihre wirtschaftliche Vormachtstellung in der Region könnte die Europäische Union zukünftig noch stärker dafür nutzen, ihren umweltschutzpolitischen Ambitionen mehr Nachdruck zu verleihen. Um eine internationale Vorreiterrolle im Bereich des Umweltschutzes einnehmen zu können, sollte die Europäische Union ihre politische Kohärenz jedoch weiter verbessern und sich ihrer eigenen Interessen, Verantwortung und Handlungsmöglichkeiten noch stärker bewusst werden. Im Laufe dieses Entwicklungsprozesses sowie bei der weiteren Ausgestaltung der politischen Agenda für den Hohen Norden sind die Bedürfnisse der indigenen Bevölkerung unbedingt zu berücksichtigen, nicht zuletzt um politische Spannungen zu vermeiden. 